\title{
Does interval laparoscopic sterilisation influence the risk of lower genital tract infections and menstrual abnormalities?
}

\author{
G Kistan, ${ }^{1}$ MB ChB, FCOG (SA), MMed; J S Bagratee, ${ }_{1}^{1}$ MB ChB, FCOG (SA), FRCOG (UK), MMed, PhD; \\ M Panday, ${ }^{2} \mathrm{MB} \mathrm{ChB}, \mathrm{FCOG}(\mathrm{SA})$ \\ ${ }^{1}$ Department of Obstetrics and Gynaecology, Nelson R Mandela School of Medicine, College of Health Sciences, University of KwaZulu-Natal, \\ Durban, South Africa \\ ${ }^{2}$ Department of Family Planning, King Dinuzulu Hospital Complex, Durban, KwaZulu-Natal, South Africa
}

Corresponding author: G Kistan (gaysheenkistan@gmail.com)

\begin{abstract}
Background. Tubal sterilisation is a safe, accessible and effective contraceptive method. There is a paucity of data regarding the risk of genital tract infections and menstrual abnormalities post sterilisation in Durban, South Africa.

Objectives. To evaluate the risk of lower genital tract infections (LGTIs) and menstrual abnormalities following interval laparoscopic sterilisation.

Methods. A prospective cohort study of 225 women undergoing sterilisation between August 2012 and April 2013, with follow-up 1 year post procedure, was conducted at King Dinuzulu Hospital, Durban.

Results. Following sterilisation, LGTIs were increased only in women with a history of infection pre sterilisation (odds ratio 6.7; 95\% CI 2.2 - 20.9; $p=0.002$ ). There was no significant risk of HIV acquisition post sterilisation. In women who had not used contraception or used barrier methods pre sterilisation, we found no significant change in menstrual patterns post sterilisation. There was an increase in menstrual bleeding and dysmenorrhoea post sterilisation among previous combined oral contraceptive users. Among women with amenorrhoea on injectable contraception pre sterilisation, $73.8 \%$ reported return to regular menses and $26.2 \%$ reported abnormal uterine bleeding post sterilisation. Among injectable contraceptive users with regular menses pre sterilisation, $71.4 \%$ reported no change in menses post sterilisation and $28.6 \%$ reported abnormal uterine bleeding post sterilisation.

Conclusion. In women undergoing interval tubal sterilisation, the risk of LGTIs was only increased in those women with a history. Menstrual abnormalities post sterilisation were more likely in women who used steroid contraception prior to sterilisation.
\end{abstract}

S Afr J Obstet Gynaecol 2017;23(2):38-42. DOI:10.7196/SAJOG.2017.v23i2.1115

Lower genital tract infections (LGTIs) manifest as abnormal vaginal discharge, pruritus, cutaneous lesions or dysuria. The aetiology is either non-sexually transmitted infections such as candidiasis, or sexually transmitted infections (STIs) (trichomoniasis, bacterial vaginosis, oral or genital herpes, chlamydia, gonorrhoea and genital warts). ${ }^{[1]}$ The prevalence of STIs in Durban, South Africa (SA) remains extremely high, with a disproportionate burden among women. STIs prevail in up to $13 \%$ of women, with an incidence rate of up to 20 per 100 women years. ${ }^{[2]}$ The sequelae include facilitated HIV transmission and acquisition, pelvic inflammatory disease (PID), infertility and chronic pelvic pain. ${ }^{[2]}$ Literature suggests that high-risk women are at an increased risk of STIs due to low rates of condom use post sterilisation. ${ }^{[3]}$ In a study of African American women residing in low-income communities, sterilised women were less likely to report using condoms with their main partners than non-sterilised women. ${ }^{[3]}$ Contrary to this finding, SangiHaghpeykar et al. ${ }^{[4]}$ reported that women at high risk of contracting STIs (a history of STIs or multiple partners) are significantly more likely to plan condom use post sterilisation with insignificant risk of exposure to disease post procedure.

Literature regarding the influence of sterilisation on the risk of HIV transmission and risk behaviours is scarce. Armstrong et al. ${ }^{[5]}$ compared HIV risk behaviours of sterilised and non-sterilised women. They found that sterilised women were more sexually active and tended not to use condoms, with only $12 \%$ of participants using condoms. No difference in HIV risk behaviour due to multiple sexual partners or prostitution was observed between the two groups, mainly due to monogamy post sterilisation. ${ }^{[5]}$

Since 1951, it has been hypothesised that sterilisation might increase a woman's risk of abnormal uterine bleeding (post tubal ligation syndrome - risk of heavy bleeding and intermenstrual bleeding) ${ }^{[6]}$ Some authors found an increased risk of menstrual abnormalities up to 5 years post sterilisation. Others found that women reported shorter or irregular menstrual cycles, decreased bleeding and dysmenorrhoea. ${ }^{[7,8]}$ Controlling for previous contraceptive use, further studies have found no direct influence of sterilisation on menstrual patterns. ${ }^{[9-11]}$

\section{Methods}

The study population included women who were referred to King Dinuzulu Hospital, Durban, SA for voluntary elective sterilisation from 1 August 2012 - 30 April 2013, and who returned for followup 1 year post procedure. Preceding the sterilisation procedure, a detailed history, gynaecological examination and an HIV test were performed on all women. Following the laparoscopic tubal sterilisation with Filshie clips, women were followed-up for 1 year post sterilisation, i.e. 1 August 2013 - 30 April 2014 on an outpatient basis. Following informed consent for participation in the study, 
women were routinely educated about safe sexual practices and offered a full gynaecological consultation and an HIV test. Women who failed to return for a follow-up consultation were contacted telephonically to return for their follow up 12 - 18 months post procedure. These women were subsequently followed-up until ethical approval expired, i.e. 25 July 2014. Women who failed to return for follow-up were excluded from the study.

Data were collected from interviews and hospital charts. Family planning registrars were trained on the use of the data sheet and the conduct of the interview. These interviews were conducted by registrars and the consultant in charge of the unit. Data were confidential and strictly for the purpose of the study.

Information regarding LGTIs, sexual behaviours and menstrual patterns was gathered by interview and women's subjective impressions. Genital tract infections (GTIs) were categorised into LGTIs (vaginal discharge, genital warts or genital ulcers) and upper genital tract infection, i.e. pelvic inflammatory disease (PID). Women who reported vaginal discharge were asked about the nature of the discharge and treatment sought to distinguish between vaginal candidiasis from STIs.

Data were captured and analysed using SPSS version 19.0 (IBM Corp., USA). Normally distributed data were analysed using a paired samples Student's $t$-test when comparing two groups. The Wilcoxon sign rank test was used on comparison of the pre- and post sterilisation differences in menstrual variables and LGTI variables. Pearson's $\chi^{2}$ or Fisher's exact tests were utilised to identify trends between categorical data variables as appropriate. A $p$-value $<0.05$ was considered as statistically significant. Permission was granted by King Dinuzulu Hospital to conduct this research study at their premises and have access to women's files. Ethical clearance was obtained from the Biomedical Research Ethics Committee of the University of KwaZulu-Natal (ref. no. BE 139/13), where the study was registered.

\section{Results}

A total of 225 women returned at 1 year post sterilisation and were included in the study cohort.

\section{Demographics}

The demographic characteristics of our cohort are shown in Table 1. The mean (SD) age of women was 35 (4.26) years. The racial profile varied; $60 \%$ were black $(n=135), 35.6 \%$ indian $(n=80), 1.8 \%$ white $(n=4)$ and $2.7 \%$ were coloured $(n=6)$.

\section{Genital tract infections (GTIs)}

Pre sterilisation, 34 women (15.1\%) reported a history of a GTI due to an STI (Table 2). Post sterilisation, 11 women (4.9\%) reported LGTIs owing to STIs. Eleven women (4.9\%) sought medical attention for a vaginal discharge post procedure. Ten women received syndromic treatment for STIs and 1 woman received treatment for vaginal candidiasis. Among the women with STIs, 10 women sought medical attention for a vaginal discharge and 1 woman for genital warts. None of these women reported a history of PID post sterilisation. Of these 11 women, 6 had previously sought medical attention for LGTIs owing to STIs pre-sterilisation (17.6\%) and 5 women $(2.6 \%)$ had no history of prior infection. There was an increase in LGTIs due to STIs post sterilisation in those women who had a history of STIs (OR 6.7; 95\% CI $2.2-20.9$; $p=0.002$ ) compared with women who had no history of infection pre sterilisation. In women with no history of a LGTI, sterilisation conferred a lower risk of LGTIs (OR 0.8 ; 95\% CI $0.7-0.9$; $p=0.001$ ).

\section{Sexual behaviour}

Most women had 1 sexual partner at the time of sterilisation ( $n=209,92.9 \%)$, with 9 women (4\%) reporting $1-3$ partners and 7 women (3.1\%) had no partners. Post sterilisation, 213 women (94.7\%) reported having 1 partner, 8 women (3.6\%) had no partner and 4 women (1.8\%) reported having 1 - 3 partners. There was no significant change in the number of sexual partners post sterilisation $(p=0.31)$.

Of the cohort, $53.3 \%(n=120)$ reported having sexual intercourse more than once weekly, $41.8 \%$ ( $n=94)$ reported weekly or monthly sexual intercourse, while $4.9 \%(n=11)$ reported not being sexually active post procedure. A total of 132 women (58.7\%) reported having the same sexual interest and pleasure compared with their experiences pre procedure, 27.1\% ( $n=61)$ had more interest and pleasure, and $14.2 \%(n=32)$ experienced less interest and pleasure.

Among the women with STIs post sterilisation, 6 (54.5\%) women had a previous history of STIs. Of these 6 women, 4 (66.7\%) were HIV-negative and 2 (33.3\%) women were HIV-positive. Among the HIV-positive women, 1 woman (50\%) reported an increase in the number of partners post procedure. Both women reported occasional and inconsistent condom use during sexual intercourse. Both women reported an increase in sexual activity post sterilisation with the same sexual interest and pleasure compared with their pre sterilisation experiences. The remaining 4 women, who were HIVnegative, all reported no condom use post sterilisation. Two of these 4 women (50\%) reported an increase in the number of sexual partners post sterilisation. Three of the 4 women (75\%) reported increased sexual activity and pleasure post sterilisation and 1 woman $(25 \%)$ reported the same sexual interest and pleasure.

Of the 5 women (45.5\%) who had no prior history of STIs pre sterilisation, all were HIV-negative. Four women (80\%) reported 1 partner pre and post sterilisation, and 1 (20\%) reported 1 partner pre sterilisation and 2 partners post sterilisation. None of

Table 1. Demographic characteristics of study cohort $(N=225)$

\begin{tabular}{ll}
\hline & Mean $(\mathrm{SD})$ \\
\hline Age (years) & $35(4.26)$ \\
Partner's age (years) & $39(5.55)$ \\
Parity $(n)$ & $3(1)$ \\
Haemoglobin $(\mathrm{g} / \mathrm{dL})$ & $12.4(6.19)$ \\
Body mass index $\left(\mathrm{kg} / \mathrm{m}^{2}\right)$ & $33.3(7.42)$ \\
SD $=$ standard deviation. &
\end{tabular}

Table 2. Genital tract infections pre and post TL

\begin{tabular}{lll}
\hline & Pre TL, $\boldsymbol{n}$ (\%) & Post TL, $\boldsymbol{n}$ (\%) \\
\hline GTIs & $34(15.1)$ & $11(4.9)$ \\
LGTI: Vaginal discharge & $30(13.3)$ & $10(4.4)$ \\
Genital warts & $2(0.9)$ & $1(0.4)$ \\
UGTI: PID & $2(0.9)$ & 0
\end{tabular}

$\mathrm{TL}=$ tubal ligation; $\mathrm{LGTI}=$ lower genital tract infection; UGTI = upper genital tract infection; PID = pelvic inflammatory disease; GTI = genital tract infection. 
these women reported condom use pre and post sterilisation. Four of these women reported more frequent sexual intercourse with the same interest and pleasure. One woman reported the same frequency, sexual interest and pleasure compared with her pre sterilisation experience.

\section{HIV status}

A total of 169 women were HIV-negative (75\%). Of the 56 women (25\%) who were HIV-positive, 38 (67.9\%) were on antiretroviral drugs (ARVs) and 18 (32.1\%) were not on treatment owing to CD4 cell counts $>350$ cells $/ \mu \mathrm{L}$. Post sterilisation, 168 women tested HIVnegative. One woman, who had tested HIV-negative pre sterilisation, had tested positive post sterilisation. She reported an increase in sexual partners, non-use of condoms and increased sexual activity post sterilisation. Among the study cohort, there was no risk of HIV acquisition post sterilisation (relative risk 0.006; 95\% CI $0.001-0.042, p=1)$. In women who were HIV-positive, the mean CD 4 cell count pre procedure was 426 cells $/ \mu \mathrm{L}$ and 601 cells $/ \mu \mathrm{L}$ post procedure. There was a significant rise in CD4 cell counts ( $p=0.02$ ), with $66.7 \%$ of HIV-positive women (38/57) being on ARVs.

\section{Contraception}

The majority of women were on injectable contraception (DepoProvera, Nur-isterate) as outlined in Fig. 1 with the remaining on combined oral contraception (COC), barrier methods or no contraception. Of the cohort, $15.5 \%(n=35)$ of women used dual contraception. Pre sterilisation, 19.1\% $(n=43)$ women used condoms occasionally, 20\% ( $n=45)$ always used condoms and $60.9 \%$ $(n=137)$ women did not use condoms during sexual intercourse. Post procedure, $37.3 \%$ of women $(n=84)$ used condoms either occasionally or always and $62.7 \%(n=141)$ did not use condoms. This increase in non-use of condoms did not reach statistical significance $(p=0.23)$.
The majority of HIV-positive women (66.7\%) always used condoms during coitus post sterilisation $(n=38)$. The remaining $21.1 \%$ of HIVpositive women $(n=12)$ used condoms occasionally and $12.3 \%(n=7)$ did not use condoms post sterilisation.

\section{Menstrual patterns}

Table 3 outlines menstrual patterns pre and post sterilisation controlling for previous contraceptive use. Women on barrier methods and those not on any contraception pre sterilisation had no statistically significant changes in their number of days of menstrual bleeding $(p=1)$ or dysmenorrhoea $(p=0.89)$.

Among the women with amenorrhoea on injectable contraception pre sterilisation, $73.8 \%(n=79)$ reported return to regular menses, and $26.2 \%(n=28)$ reported abnormal uterine bleeding post sterilisation (prolonged bleeding, heavy bleeding and cycle irregularity). Among injectable contraceptive users with regular menses or spotting pre sterilisation, $71.4 \% \quad(n=30)$ reported no change in menses post sterilisation and 28.6\% ( $n=12)$ reported abnormal uterine bleeding

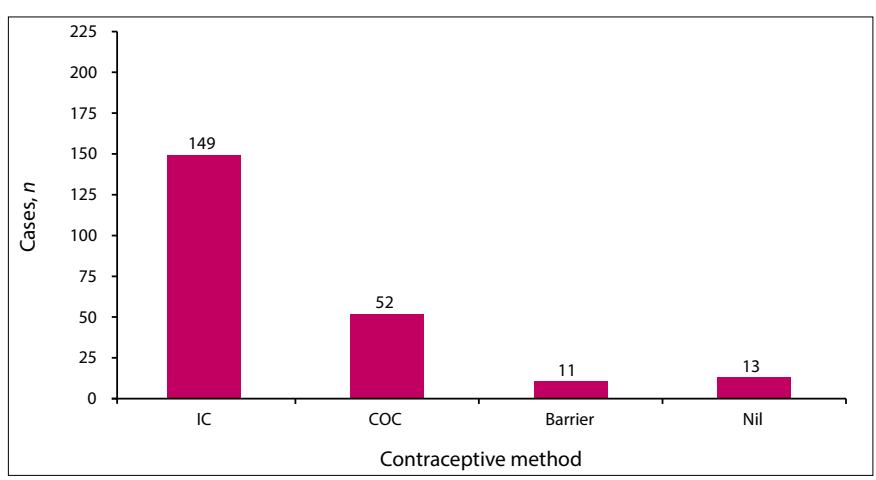

Fig. 1. Contraceptive use pre sterilisation. (IC = injectable contraceptive; $C O C=$ combined oral contraceptive).

Table 3. Menstrual patterns pre and post TL controlling for contraceptive use

\begin{tabular}{|c|c|c|c|c|c|c|c|c|}
\hline \multirow[b]{2}{*}{ Parameter } & \multicolumn{2}{|c|}{ IC $(N=149)$} & \multicolumn{2}{|c|}{$\operatorname{COC}(N=52)$} & \multicolumn{2}{|c|}{ Barrier $(N=11)$} & \multicolumn{2}{|c|}{ Nil $(N=13)$} \\
\hline & Pre TL & Post TL & Pre TL & Post TL & Pre TL & Post TL & Pre TL & PostTL \\
\hline \multicolumn{9}{|l|}{ Days of menses, $n(\%)$} \\
\hline Amenorrhoea & $107(71.8)$ & 0 & 0 & 0 & 0 & 0 & 0 & 0 \\
\hline$<4$ & $27(18.1)$ & $79(53.0)$ & $38(73.1)$ & $4(7.7)$ & $7(63.6)$ & $7(63.6)$ & $8(61.5)$ & $69(46.2)$ \\
\hline $4-8$ & $14(9.4)$ & $65(43.6)$ & $14(26.9)$ & $44(84.6)$ & $3(27.3)$ & $3(27.3)$ & $5(38.5)$ & $7(53.8)$ \\
\hline$>8$ & $1(0.7)$ & $5(3.4)$ & 0 & $4(7.7)$ & $1(9.1)$ & $1(9.1)$ & 0 & 0 \\
\hline \multicolumn{9}{|l|}{ Flow of menses, $n(\%)$} \\
\hline Amenorrhoea & $107(71.8)$ & 0 & 0 & 0 & 0 & 0 & 0 & 0 \\
\hline Light & $21(14.1)$ & $46(30.9)$ & $24(46.2)$ & $2(3.9)$ & $4(36.4)$ & $4(36.4)$ & $4(30.8)$ & $3(23.1)$ \\
\hline Average & $18(12.1)$ & $76(51.0)$ & $28(53.9)$ & $27(51.9)$ & $5(45.5)$ & $4(36.4)$ & $6(46.1)$ & $7(53.9)$ \\
\hline Heavy & $3(2.0)$ & $27(18.1)$ & 0 & $23(44.2)$ & $2(18.2)$ & $3(27.3)$ & $3(23.1)$ & $3(23.1)$ \\
\hline Amenorrhoea & $107(71.8)$ & 0 & 0 & 0 & 0 & 0 & 0 & 0 \\
\hline Regular & $32(21.5)$ & $130(87.3)$ & $52(100)$ & $47(90.4)$ & $10(90.9)$ & $10(90.9)$ & $13(100)$ & $13(100)$ \\
\hline Irregular & $10(6.7)$ & $19(12.8)$ & 0 & $5(9.6)$ & $1(9.1)$ & $1(9.1)$ & 0 & 0 \\
\hline \multicolumn{9}{|l|}{ Pain with menses, $n(\%)$} \\
\hline Nil & $149(100)$ & $104(69.8)$ & $52(100)$ & $19(36.5)$ & $11(100)$ & $11(100)$ & $10(76.9)$ & $9(9.2)$ \\
\hline Yes, not requiring meds & 0 & $39(26.2)$ & 0 & $28(53.9)$ & 0 & 0 & $3(23.1)$ & $3(23.1)$ \\
\hline Yes, requiring meds & 0 & $6(4.0)$ & 0 & $5(9.6)$ & 0 & 0 & 0 & $1(7.7)$ \\
\hline
\end{tabular}


post sterilisation (prolonged bleeding and heavy menstrual bleeding). Among previous injectable users, there was a significant increase in days of bleeding $(p=0.03)$, amount of menstrual bleeding $(p=0.01)$ and dysmenorrhoea post sterilisation $(p=0.005)$. Fourteen women (9.4\%) sought medical attention post sterilisation for heavy menses $(n=4,28.6 \%)$ and cycle irregularity $(n=10,71.4 \%)$. These women were assessed clinically for abnormalities and 1 woman (7.1\%) was found to have leiomyomas and was referred to her base hospital for further treatment. The remaining women in whom no abnormalities were found were successfully treated medically.

Among previous COC users, 37 women (71.2\%) reported heavy or prolonged menses post sterilisation. Cycle irregularity was reported by 5 women $(9.6 \%)$ and dysmenorrhoea by 33 women $(63.5 \%)$. An increase in duration of menses $(p=0.02)$, flow of menses $(p=0.01)$ and dysmenorrhoea $(p=0.001)$ was found in previous COC users post sterilisation. Five women (9.6\%) sought medical attention post sterilisation for heavy menses and cycle irregularity. Clinically, no organic cause was found and these women were successfully treated.

\section{Discussion}

In SA, female sterilisation accounts for up to $14 \%$ of contraceptive use among sexually active women. ${ }^{[12]}$ In our setting with a high prevalence of STIs and HIV, ${ }^{[2]}$ there are concerns regarding the impact of sterilisation on the spread of STIs and HIV. We found the risk of LGTIs owing to STIs was not directly affected by interval tubal sterilisation but is rather a reflection of sexual behaviour prior to sterilisation. A history of a prior STI in women undergoing tubal sterilisation conferred a 6-fold risk of a repeat STI after sterilisation compared with women who had no history of infection pre sterilisation. In this subset of women, non-use of condoms post sterilisation, increase in sexual partners and sexual activity, interest and pleasure was found. There was no risk of HIV acquisition post sterilisation; however, an increase in STIs and HIV risk behaviour was found in women with a past history of STIs. Similarly, low rates of condom use in high-risk groups post sterilisation is supported by previous studies. ${ }^{[3]}$ Women in our cohort were unlikely to use condoms if they were HIV-negative or reported just one sexual partner post sterilisation. Previous literature found that women with a past history of STIs are more likely to plan condom use post sterilisation, ${ }^{[4]}$ which differs from our study findings. In women with a past history of STIs, interventions to increase condom use post sterilisation are needed to reduce both the risk of subsequent infections and HIV. Education of both women and partners and offering ample free supplies may alleviate this problem. Sweat et al., ${ }^{[13]}$ found condom social marketing (condom branding, media-based marketing campaign and targeted population-based campaigns) may double the odds of condom use. This may be an effective strategy in high-risk populations to advocate for and increase condom use.

Reassuring findings from our study were that the majority of HIVpositive women used condoms post sterilisation (66.7\%), which may reflect women's use of the health system and counselling at HIV clinics which needs to be emphasised and extended to all health facilities for both HIV-positive and -negative women.

In women with no prior history of STIs, we found no risk of infection post sterilisation. This was due to the older age of the women, monogamous relationships, no change in partners post sterilisation and no change in condom use post sterilisation. Furthermore, consistent with previous studies, ${ }^{[14]}$ the majority of women reported no change in sexual interest and pleasure after interval tubal sterilisation (58\%).
In women who were not on steroid contraception pre sterilisation, we found no change in menstrual patterns post sterilisation. This is supported by Wilcox et al., ${ }^{[7]}$ who found that menstrual abnormalities (dysmenorrhoea, heavy bleeding) are more common 5 years post sterilisation than within the first 2 years post procedure. Menstrual abnormalities in our study cohort were significant in women who previously used steroid contraception. There was an increase in the duration and amount of menstrual bleeding and dysmenorrhoea post sterilisation among previous COC users. Among previous injectable contraceptive users, abnormal uterine bleeding and dysmenorrhoea was found in our study cohort. Our findings suggest that menstrual changes post sterilisation are due to recent discontinuation of steroid contraception, rather than the sterilisation procedure itself. The literature further supports that sterilisation has no direct influence on menstrual patterns. ${ }^{[9-11]}$ Accounting for prior contraceptive use, changes in menses post sterilisation have been shown to result in the return of pre-contraceptive use characteristics, including dysmenorrhoea, heavy menstrual flow and cycle irregularity. ${ }^{[10,11]}$ Previously, it has been shown that women on injectable contraception have re-establishment of menstrual cycles up to 6 - 8 months following the last injection. ${ }^{[15]}$ Women in our cohort subjectively reported changes in menses due to prolonged amenorrhoea (women on injectable contraception) and due to previous COC use.

\section{Study limitations}

Selection bias was encountered in that only women who were willing and able to present themselves one year post sterilisation were included in the study cohort. A further limitation was that data pertaining to menstrual patterns and the presence of STIs were obtained from the women's history and from subjective impressions.

\section{Conclusions}

The risk of LGTIs due to STIs in women undergoing tubal ligation was increased only in those women with a history of infection pre sterilisation. Women with a history of STIs need to be counselled and offered follow-up visits to allow for screening for infection post procedure. They need to be counselled on the correct and consistent use of condoms post sterilisation. In women who had used steroid contraception previously, menstrual abnormalities were more likely post sterilisation, which highlights the importance of counselling these women prior to sterilisation.

Further research on the correlations between HIV, sterilisation and risk behaviour is needed. Large trials examining risk behaviours in HIVpositive and HIV-negative women post sterilisation is recommended.

Acknowledgements. We would like to acknowledge Ms Jesca Batidzirai, statistician at the University of KwaZulu-Natal, for her assistance with the statistical analysis of the data. We wish to express our gratitude to the management and staff of King Dinuzulu Hospital for permitting us to conduct our study at their institution and to the women from King Dinuzulu Hospital who participated in our study.

Author contributions. GK designed the study with input from JB and MP. MP monitored data collection and GK collected data for analysis. GK, JB and MP analysed and interpreted the data and together prepared the manuscript. All authors contributed to and approve the manuscript.

Funding. None.

Conflicts of interest. None. 
1. Landers DV, Wiesenfeld HC, Heine RP, Krohn MA, Hillier SL. Predictive value of the clinical diagnosis of lower genital tract infection in women. Am J Obstet Gynecol 2004:190(4):1004-1008. https://doi.org/10.1016/j.ajog.2004.02.015

2. Naidoo $\mathrm{S}$, Wand $\mathrm{H}$, Abbai $\mathrm{S}$, Ramjee G. High prevalence and incidence of sexually transmitted infections among women living in Kwazulu-Natal, South Africa. AIDS Res Ther 2014;11(31):2-7. https://doi.org/10.1186/1742-6405-11-31

3. Semaan S, Lauby J, Walls C. Condom use with main partners by sterilized and non-sterilize Semaan S, Lauby J, Walls C. Condom use with main partners by sterilized
women. Wom Health 1997;25(2):65-85. https://doi.org/10.1300/J013v25n02_04

4. Sangi-Haghpeykar H, Poindexter A. Planned condom use among women undergoing tubal sterilisation. Sex Transm Dis 1998;25(7):335-341. https://doi.org/10.1097/00007435-199808000-

5. Armstrong KA, Samost L, Tavris DR. HIV-risk behaviors of sterilized and nonsterilized women in drug-treatment programmes-Philadelphia, 1989-1991. MMWR 1992;41(9):149-152

6. Peterson HB, Pollack AE, Warshaw S. Tubal Sterilization. Chapter 23. In: Rock JA, Jones HW, Te Linde RW, eds. Te Linde's Operative Gynaecology. Philadelphia: Wolters Kluwer Health/Lippincot Williams \& Wilkins, 2008:609-629.

7. Wilcox LS, Martinez-Schnell B, Peterson HB, Ware JH, Hughes JM. Menstrual function after tuba sterilization. Am J Epidemiol 1992;135(12):1368-1381. https://doi.org/10.1093/oxfordjournals.aje. al16248

8. Peterson HB, Jeng G, Folger S, Hillis S, Marchbanks P, Wilcox L. The risk of menstrual abnormalities NEJM200012073432303
9. Harlow B, Missmer SA, Cramer DW, Barbieri RL. Does tubal sterilization influence the subsequent risk of menorrhagia or dysmenorrhea? Fertil Steril 2002;77(4):754-760. https://doi.org/10.1016/ S0015-0282(01)03253-8

10. Westhoff C. Tubal sterilization - safe and effective. N Engl J Med 2000;343:1724-1726. https://doi. org/10.1056/NEJM200012073432310

11. Rulin MC, Davidson AR, Philliber SG, Graves WL, Cushman LF. Long-term effect of tubal sterilization on menstrual indices and pelvic pain. Obstet Gynecol 1993;82(1):118-121.

12. South African Demographic and Health Survey. 2003. http://www.gov.za/documents/south-africandemographic-and-health-survey-sadhs (accessed 2 December 2015).

13. Sweat MD, Denison J, Kennedy C, Tedriwc V, O'Reilly K. Effects of condom social marketing on condom use in developing countries: A systematic review and meta-analysis, 1990 - 2010. Bull World Health Organ 2012;90(8):613-622. https://doi.org/10.2471/BLT.11.094268

14. Costello C, Hillis S, Marchbanks P, Jamieson D, Peterson H. The effect of interval tubal sterilization on sexual interest and pleasure. Obstet Gynaecol 2002;100(3):511-517. https://doi.org/10.1016/ S0029-7844(02)02042-2

15. Westhoff C. Depot-medroxyprogesterone acetate injection (Depo-Provera): A highly effective contraceptive option with proven long-term safety. Contraception 2003;68(2):75-87. https://doi. org/10.1016/S0010-7824(03)00136-7 